\title{
Survival rate of cervical cancer: A five year review at the national center for radiotherapy and nuclear medicine, Korle-Bu Teaching Hospital, Accra. Ghana
}

\author{
Opoku SY ${ }^{*}$, Yarney $J^{2}$, Vanderpuye $V^{2}$, Koranteng $I^{2}$, Kyei-Adesi $K^{1}$, Antwi WK ${ }^{1}$ and Donkor $A^{2}$ \\ ${ }^{1}$ Department of Radiography, School of Biomedical and Allied Health Sciences, University of Ghana, Ghana \\ ${ }^{2}$ National Center for Radiotherapy and Nuclear Medicine, Korle Bu Teaching Hospital, Ghana
}

\begin{abstract}
Background: Worldwide, cervical cancer is the second most deadly cancer, causing more than 273,000 deaths each year. Cervical cancer accounts for $2.1 \%$ of all deaths and $9 \%$ of female cancer deaths. The International Agency for Cancer Research estimated that in 2013, 3038 Ghanaian women developed cervical cancer and more than $80 \%$ Ghanaian women died of the disease. This high mortality is due to lack of information and knowledge as well as inadequate diagnoses and treatment. It was estimated that 6.67 million women over the age of 15 were currently at risk of developing cervical cancer in Ghana
\end{abstract}

Aim: The study aimed at determining the five - year survival rate of cervical cancer patients who received radiotherapy at the National Center for Radiotherapy and Nuclear Medicine (NCRNM), Korle-Bu Teaching Hospital, Accra. Ghana.

Methods: A quantitative retrospective cohort design was used for this study. Medical records of cervical cancer patients who received radiotherapy in 2007 were obtained from the records office. Descriptive statistics was used to determine frequencies and percentages of tumour stage, age and other factors and their effect on the overall survival rates of patients.

Results: A total of 100 cervical cancer patients were followed and 76 responded. Forty-one percent (41\%) five- year survival rate was noted in the study. It was further noted that patients who received radical radiotherapy recorded $86.7 \%$ survival rate.

Conclusion: The age of the patients, the tumor stage, and the type of treatment used influenced the survival rate of patients.

\section{Introduction}

Worldwide, cervical cancer is the second most deadly cancer, causing more than 273,000 deaths each year. Cervical cancer accounts for $2.1 \%$ of all deaths and $9 \%$ of female cancer deaths [1-4]. The age standardized mortality rate for cervical cancer in Ghana, West Africa is more than three times the global cervical cancer mortality rate (27.6/100,000 versus 7.8/100,000 respectively). The World Health Organization (WHO) predicts that by the year 2025, 5,000 new cases of cervical cancer and 3,361 cervical cancer deaths will occur annually in Ghana. As a result of the absence of national cancer registry in Ghana, the actual incidence of cervical cancer is unknown. However, conservative estimates put the cervical cancer as the leading female cancer ahead of breast cancer in the country. The International Agency for Cancer Research estimated that in 2013, 3038 Ghanaian women developed cervical cancer and more than $80 \%$ Ghanaian women died of the disease. This high mortality is due to lack of information and knowledge as well as inadequate diagnoses and treatment. It was estimated that 6.67 million women over the age of 15 were currently at risk of developing cervical cancer in Ghana $[3,4]$.

Several studies have shown that the incidence of cervical cancer, as well as survival and mortality, vary with ethnic group and socioeconomic status. For instance, studies have demonstrated higher incidence of cervical cancer in Hispanic and Black Women than in White Women [5-7] and that incidence of cervical cancer is highest in women with the lowest socio-economic status [8]. However, studies have shown that $30 \%$ of cervical cancers are preventable [1] and about a third of them being potentially curable upon early detection and proper diagnosis.

Treatment modalities for cervical cancer include surgery, chemotherapy and radiotherapy (both external beam radiotherapy and brachytherapy). A combination of these treatment modalities called adjuvant therapy could be used in certain instances depending on the stage of the disease [1]. Unlike most cancers, cervical cancer has a central causal factor, which is the Human Papilloma Virus (HPV) as posited by Franco and colleagues [9]. The Papanicoloau (Pap) screening test is currently the most widely used diagnostic tool in detecting the presence of cervical cancer [10]. The huge disparities in morbidity and mortality between developed and developing countries exist largely because over the last few decades, developed countries have implemented effective programmes for the prevention and early detection of cervical cancer. In some countries, the incidence and mortality rate have reduced by up to $80 \%$ [11].

Correspondence to: Dr. Samuel Y. Opoku, Department of Radiography, School of Biomedical and Allied, Health Sciences, College of Health Sciences, University of Ghana, Korle Bu. Accra. Ghana, E-mail: syopoku@chs.edu.gh

Received: May 03, 2016; Accepted: June 24, 2016; Published: June 29, 2016 
The effects of factors such as tumour stage and age of patient on the overall survival rates of patients are not known in Ghana. Patients presenting with cervical cancer at the National Center for Radiotherapy and Nuclear Medicine (NCRNM), Korle-Bu Teaching Hospital, have little information on their chances of survival. There was the need to ascertain the survival rate of patients undergoing radiotherapy. The study therefore aimed at investigating the five year survival rate of cervical cancer patients who received radiotherapy as part of their treatment at the Center.

\section{Method}

Using a quantitative retrospective cohort research design, data was gathered from cervical cancer patients treated at the National Center for Radiotherapy and Nuclear Medicine, Korle-Bu Teaching Hospital, Accra in 2007. The Hospital is the largest and leading referral health facility in Ghana. The study included Ghanaian patients who presented with cervical cancer and completed their treatment.

Data collection was done between April 2014 and May 2014. Medical records of cervical cancer patients were obtained from the records office. Patients' information, diagnosis, the date of diagnosis, tumour grade, tumour stage, start date of treatment, and contact details were obtained. The status (dead or alive) of the patients was obtained through telephone conversation and inputted in the data collection sheet.

Ethical approval was obtained from Ethical and Protocol Review Committee School of Biomedical and Allied Health Sciences, University of Ghana. Written permission was obtained from the Management of National Center for Radiotherapy and Nuclear Medicine. Participants were informed about the confidentiality of the study through telephone conversations before further questions were asked. Patient identity was kept confidential throughout the study due to the sensitive nature of the study.

\section{Results}

One hundred (100) cervical cancer patients with histologically proven stage of the disease were retrospectively reviewed and followedup. Seventy-six percent of the patients were accessible. The remaining $24 \%$ of the patients were unreachable because of challenges of reaching them through the phone contacts provided. The age range of the participants was between 30-90 years. The total of 56 patients received radical radiotherapy whilst the remaining 20 patients received palliative radiotherapy.

\section{Discussion}

This study is the first to be conducted to assess the five-year survival rate of cervical cancer patients after receiving radiotherapy in Ghana. The findings of the study reveal exceptional knowledge on survival rate of cervical cancer patients. Forty-one percent (41\%) five-year survival rate was identified. The stage of the disease, intent of radiotherapy, and age of the patient at diagnosis were three major contributing factors to survival after radiotherapy [12].

The finding of the study showed that the five year survival rate among patients who presented with stage I of the disease was as high. On the other hand, rate for patients with stage IV was as low as $25 \%$. These findings are consistent with Patra's report [13] which states that when cervical cancer is found in the early stages, it can be treated and cured. However, because of lack of knowledge and public awareness on cervical cancer, and lack of access to timely and efficient healthcare, most Ghanaian cervical cancer patients present to the radiotherapy department with late stage disease.

Radiotherapy treatment can take two approaches; the palliative approach in which the aim of the treatment is to manage the symptoms and the radical treatment approach aimed at total cure of the patient. The stage and age at which a patient presents may determine the kind of treatment approach that will be used. It was also identified that radical treatment was the major aim and accounted for $74 \%$ while palliative accounted for $26 \%$. This is because majority of the patients presented with a stage II disease. The respondents who received palliative care had a $25 \%$ survival rate while those on radical treatment had $86.7 \%$ survival rate [14].

A study by the Office of National Statistics in the United Kingdom revealed that the five-year relative survival rates for cervical cancer in England during 2005-2009 ranged from 89\% in 15-39 year olds, 79\% in $40-49$ year olds, $65 \%$ in $50-59$ year olds, $55 \%, 42 \%$ and $22 \%$ in 60 $69,70-79$ and $80-99$ year olds respectively [14]. Our results greatly differ from the Office of National Statistics findings. Our study showed that $25 \%$ survival in $30-39$ year olds was $57.9 \%$; $44.4 \%$; $37.5 \%$; $28.6 \%$ and $0 \%$ survival in 40-49,50-59, 60-69 and 70-79, 80-89 year-groups respectively. The lower survival for the aged could be due to the late stage of diagnosis and also co-morbidities associated the old age. Also, it could be due to the lack of supportive care associated with the Ghanaian healthcare system [15].

The findings of the study must be interpreted with caution. This is because patients' records were manually kept at the Radiotherapy Department resulting in data misplacement. This made it difficult to access important information such as date of disease diagnosis, contacts, and treatment intent. Also, some of the relations of the patients who were contacted were reluctant and uncooperative in disclosing the survival status of the patient.

\section{Conclusion}

Patients treated at the radiotherapy department of the Korle- $\mathrm{Bu}$ Teaching Hospital in 2007 have $41 \%$ survival rate compared to the USA, which is about 59\% in African Americans and 69\% in Hispanics. The overall survival rate was influenced by the age and stage at which patients presented. The poor rate of survival compared to other countries could be attributed to delayed presentation because of lack of access to screening, and poor awareness of the disease. Also, the absence of appropriate follow-up structure after the cervical cancer treatment may have influenced their life negatively. Going forward, electronic way of keeping patients information should be implemented to eradicate misplacement of the folders. The centre should institutionalized appropriate follow-up structures to assess the quality of life of cancer patients after radiotherapy. The government and non-governmental organizations should intensify public education on cervical cancer. Similarly, cervical cancer screening policy should be introduced.

\section{References}

1. World Health Organization (2008) The silent crisis of cancer in the developing world WHO Press.

2. Kumar V, Abbas AK, Fausto N, Mitchell RN(2007) Robbins basic pathology (8t edition). Saunders Elsevier.

3. Yang BH, Bray FI, Parkin DM, Sellors JW, Zhang ZF (2004) Cervical cancer as a priority for prevention in different world regions: an evaluation using years of life lost. Int $J$ Cancer 109: 418-424. [Crossref]

4. Murthy P, Li E, Azzam H, Narasimhadevara A, Yezzo M (2010) Cervical Cancer Mortality: A Preventable Tragedy. In: Murthy PaS, C., editor. Women's Global Health 
and Human Rights. Sudbury: Jones and Bartlett Publishers

5. World Health Organization Report (2002) Cervical cancer screening in developing countries: report of the World Health Organization consultation.

6. Centre for Disease Control (2012) Human Papilloma Virus Associated Cancers in the United States, 2004-2008. MMWR 61: 258-261.

7. Clegg L, Reichmann M, Miller B (2008) Impact of socioeconomic status on cancer incidence and stage at diagnosis: selected findings from the surveillance, epidemiology, and end results: national longitudinal mortality study. Cancer Causes and Control, 20: 417-35.

8. Patel NR, Rollison DE, Barnholtz-Sloan J, Mackinnon J, Green L, et al. (2009) Racia and ethnic disparities in the incidence of invasive cervical cancer in Florida. Cancer 115: 3991-4000. [Crossref]

9. Clegg-Lamptey J, Dakubo J, Attobra YN (2009) Why do breast cancer patients report late or abscond during treatment in ghana? A pilot study. Ghana Med J 43: 127-131. [Crossref]
10. Franco E, Duarte-Franco E, Ferenczy A (2001) Cervical cancer: epidemiology, prevention and the role of human papilloma virus, Canadian Medical Association Journal 1017-1023.

11. Domfeh A, Wiredu E, Adjei A, Ayeh-Kumi P, Adiku T, et al. (2008) Cervical human papillomavirus infection in accra, ghana. Ghana Med J 42: 71-78. [Crossref]

12. Lipnick RL, Cotruvo JA, Hill RN, Bruce RD, Stitzel KA, et al. (1987) Trends in mortality from cervical cancer in the Nordic countries: association with organised screening programmes. Lancet 1: 1247-1249. [Crossref]

13. World Health Organization (2007) Information centre on HPV and cervical cance (HPV Information Centre): summary report on HPV and cervical cancer statistics in Ghana.

14. Patra S, Panda D (2010) Cervical cancer screening in developing countries. Indian J Cancer 47: 344-345. [Crossref]

15. The Office for National Statistics (2010) Cancer survival in England: patients diagnosed from 2005-2009 and followed-up to London: ONS 2011.

Copyright: (C2016 Goonewardene SS. This is an open-access article distributed under the terms of the Creative Commons Attribution License, which permits unrestricted use, distribution, and reproduction in any medium, provided the original author and source are credited. 